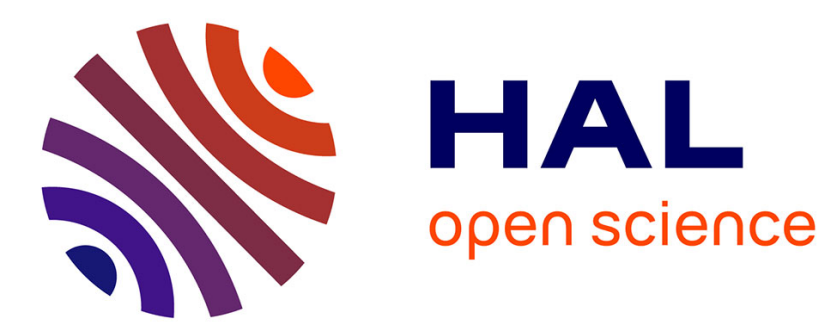

\title{
Pour un moment épistémologique du droit - constitutionnel
}

\author{
Xavier Magnon
}

\section{To cite this version:}

Xavier Magnon. Pour un moment épistémologique du droit - constitutionnel. Annuaire internationale de justice constitutionnelle, 2016, pp.13-25. hal-01448564

\section{HAL Id: hal-01448564 \\ https://hal.science/hal-01448564}

Submitted on 26 Feb 2018

HAL is a multi-disciplinary open access archive for the deposit and dissemination of scientific research documents, whether they are published or not. The documents may come from teaching and research institutions in France or abroad, or from public or private research centers.
L'archive ouverte pluridisciplinaire HAL, est destinée au dépôt et à la diffusion de documents scientifiques de niveau recherche, publiés ou non, émanant des établissements d'enseignement et de recherche français ou étrangers, des laboratoires publics ou privés. 
Institut Louis Favoreu

Groupe d'Études et de Recherches

comparées sur la Justice Constitutionnelle

Équipe associée au CNRS (UMR7318)

Aix-en-Provence

\title{
Annuaire \\ International \\ de Justice \\ Constitutionnelle
}

\author{
$\mathrm{XXXI}$ \\ 2015 \\ (extraits)
}

ECONOMICA

49, rue Héricart

75015 Paris
PRESSES UNIVERSITAIRES

D'AIX-MARSEILLE

3, Avenue R. Schuman

13628 Aix-en-Provence cedex 01

2016 
ÉTUDES 


\title{
DE LA POSSIBILITÉ D'UN DISCOURS CONSTITUTIONNEL CRITIQUE
}

\author{
Études rassemblées par Xavier Magnon
}

\begin{abstract}
Pour un moment épistémologique du droit - constitutionnel par Xavier MAGNON, Professeur à l'université de Toulouse 1 Capitole, Institut Maurice Hauriou
\end{abstract}

Il n'y a de science que critique

par Bernard LAHIRE, Professeur de sociologie, École normale supérieure de Lyon, Membre senior de l'Institut universitaire de France

La possibilité d'une analyse du droit (constitutionnel) scientifiquement et juridiquement critique par Régis PONSARD, Maître de conférences en droit public, université de Champagne, Centre d'études des normes juridiques Yan Thomas, École des hautes études en sciences sociales

Les moyens d'une analyse scientifiquement et juridiquement critique : l'exemple de l'étude des décisions du Conseil constitutionnel

par Régis PONSARD, Maître de conférences en droit public,

université de Champagne, Centre d'études des normes juridiques Yan Thomas, École des hautes études en sciences sociales 


\title{
POUR UN MOMENT ÉPISTÉMOLOGIQUE DU DROIT - CONSTITUTIONNEL -
}

\author{
par Xavier MAGNON
}

«La science, dans son besoin d'achèvement comme dans son principe, s'oppose absolument à l'opinion. S'il lui arrive, sur un point particulier, de légitimer l'opinion, c'est pour d'autres raisons que celles qui fondent

l'opinion ; de sorte que l'opinion a, en droit, toujours tort. L'opinion pense mal; elle ne pense pas : elle traduit des besoins en connaissances. En désignant les objets par leur utilité, elle s'interdit de les connaître. On ne peut rien fonder sur l'opinion: il faut d'abord la détruire. Elle

est le premier obstacle à surmonter. (...) L'esprit scientifique nous

interdit d'avoir une opinion sur des questions que nous ne comprenons pas, sur des questions que nous ne savons pas formuler clairement. Avant tout, il faut savoir poser des problèmes. Et quoi qu'on dise, dans la vie scientifique, les problèmes ne se posent pas d'eux-mêmes. C'est précisément ce sens du problème qui donne la marque du véritable esprit scientifique.

Pour un esprit scientifique, toute connaissance est une réponse à une question. S'il n'y a pas eu de question, il ne peut y avoir connaissance scientifique. Rien ne va de soi. Rien n'est donné. Tout est construit."

G. Bachelard, La formation de l'esprit scientifique, Vrin, Bibliothèque des textes philosophiques, 2011, p. 16

«Si on sait ce que l'on fait, on le fait mieux: c'est le passage d'une pratique à une méthode. (...) le métier est quelque chose qui existe à

l'état pratique, on peut faire des choses magnifiques sans avoir de métadiscours sur sa pratique. (...) Néanmoins, le métier est une condition nécessaire mais pas suffisante. On fait d'autant mieux son métier qu'on le mâ̂trise sur le plan conscient, qu'on est capable de porter

à l'explication les principes pratiques qu'on met en æuvre dans sa pratique, qu'on a transformé en règles des schèmes, posé des règles qui

peuvent devenir des règles collectives et être utilisées même par des adversaires comme un rappel à l'ordre. La codification a une importance considérable. L'épistémologie, c'est la codification d'un métier, ce qui lui fait subir une transmutation: on passe à un autre ordre quand on fait sciemment ce qu'on fait. "

P. Bourdieu, Sur l'État. Cours du Collège de France 1989-1992, Seuil, Raisons d'agir, Cours et travaux, 2012, p. 152.

Nul constitutionnaliste ne doute, à juste titre sans doute, de proposer une lecture critique de son objet d'étude. Il n'est cependant pas certain qu'une réflexion existe sur les conditions même d'un tel discours. Un discours critique sera entendu comme un discours sceptique vis-à-vis de son objet d'étude, qui met et remet constamment en question ce qu'il observe afin d'en avoir une meilleure connaissance 
dans ce qu'il a de visible mais, surtout, dans ce qu'il cache. Ainsi entendue, la dimension critique du discours du constitutionnaliste est déjà moins nette. Il n'en demeure pas moins, quelle que soit l'ambition critique du discours sur le droit constitutionnel, que la possibilité d'un discours critique présuppose l'existence d'un cadre conceptuel et méthodologique à partir duquel sera observé ce qui est étudié. Dans le sens usuel du terme, le juriste propose un discours critique; dans le sens scientifique, à défaut d'avoir posé les conditions même de son discours critique, ce discours ne saurait l'être. Autrement dit, la démarche critique du constitutionnaliste ne saurait être retenue que dans une signification usuelle et non pas dans une signification scientifique pour reprendre la distinction établie par Bachelard à propos $\mathrm{du}$ «conflit des significations $»^{1}$. Pour pouvoir tenir un discours critique, il est nécessaire de savoir ce que l'on fait. La position générale qui sera défendue consiste à soutenir qu'un discours critique n'est possible que pour autant que celui-ci repose sur un cadre conceptuel et méthodologique conscient et formalisé.

En France, la réflexion épistémologique en droit n'est certes pas originale ${ }^{2}$, mais elle présente sans doute deux défauts au regard de la perspective que nous entendons adopter ici. En premier lieu, elle est une question laissée aux théoriciens du droit et n'est ainsi pas, peu ou mal diffusée à l'ensemble de la doctrine. À demeurer dans le cadre des seuls spécialistes, elle peine à montrer la portée pratique décisive que doit avoir la réflexion épistémologique. En second lieu, cette réflexion épistémologique se contente souvent de rendre compte des possibles, des conditions de la connaissance en général, selon les différents courants, ce qui est à l'évidence nécessaire, mais sans véritablement poser un programme épistémologique ${ }^{3}$. Elle ne prend pas position. Elle ne défend pas une épistémologie propre, c'est-à-dire les conditions minimales d'une connaissance scientifique spécifique du droit ${ }^{4}$.

Telle est précisément l'ambition de ce dossier que de mettre en évidence les questions qu'impose le choix d'une épistémologie propre à la science juridique, accessible, et d'en montrer la portée pratique décisive pour l'ensemble de la discipline juridique. Comment faut-il connaître le droit ? Quelles règles le discours sur le droit doit-il s'imposer pour être scientifique? Qu'est-ce que « faire du droit », à savoir tenir un discours scientifique sur cet objet?

L'opportunité d'une telle réflexion semble devoir s'imposer aujourd'hui de manière naturelle en droit constitutionnel. En France, il est possible de considérer de

G. BACHELARD, Le rationalisme appliqué, Puf, 1949, p. 148.

Voir en particulier les notes suivantes.

Tel est le cas en particulier des deux ouvrages de référence en français sur la question : C. ATIAS, Epistémologie juridique, Dalloz, Précis, $1^{\mathrm{re}}$ éd., 2002, 230 p.; V. CHAMPEIL-DesPlats, Méthodologies $d u$ droit et des sciences $d u$ droit, Dalloz, Méthodes du droit, 2014, 432 p.

4 Voir cependant et par exemple : A. VIALA qui propose de répondre à la question « comment connaître le droit? » en écartant les deux obstacles épistémologiques que sont l'idéalisme et l'empirisme pour proposer une voie médiane : la construction d'une théorie du droit (Philosophie du droit, Ellipses, Cours magistral, 2010, $2^{\mathrm{e}}$ partie de l'ouvrage, p. 133 et s.).

Voir également pour une défense du caractère décisif de disposer d'une épistémologie : A. GESLIN, "L'importance de l'épistémologie pour la recherche en droit ", in La recherche juridique vue par ses propres acteurs, Actes de colloque IFR n ${ }^{\circ} 23$, Presses de l'Université Toulouse 1 Capitole, 2016, pp. 79-130.

Voir encore pour des ouvrages dont l'intitulé s'inscrit dans une démarche prescriptive: P. DuboucheT, Trois essais pour une théorie générale du droit. Science, épistémologie et philosophie du droit, L'Hermès, Philosophie du droit, 1998, 80 p.; J.-F. PERrin, Pour une théorie de la connaissance juridique, Librairie Droz, Genève, 1979, $1^{\text {re }}$ éd., 177 p.

Voir enfin et surtout, défendant une épistémologie du droit particulière, l'approche pluridimensionnelle du droit: R. PONSARD, Les catégories juridiques et le Conseil constitutionnel. Contribution à l'analyse du droit et du contentieux constitutionnel, Université Paris 1 Panthéon-Sorbonne, 8 décembre 2011, Thése dactyl., 606 p. La lecture proposée dans ses lignes doit beaucoup à ces travaux et aux discussions (sans fin) nourries avec Régis Ponsard. 
manière schématique que nous nous situons dans une troisième phase de la doctrine constitutionnaliste moderne. Le discours sur le droit constitutionnel s'est d'abord détaché de la «science politique » pour se centrer sur les normes juridiques plutôt que sur la pratique institutionnelle (de l'étude des régimes politiques à celle des Constitutions), avant de se tourner, par mimétisme et conservatisme juridique mais aussi pour des raisons historiques liées à la naissance de la justice constitutionnelle, vers l'interprétation de ces normes par le juge, pour embrasser aujourd'hui, et retrouver sans doute également ${ }^{5}$, des questionnements plus conceptuels. Telle est la marque contemporaine du discours constitutionnel tel qu'on le retrouve dans la production de nombreuses thèses en droit constitutionnel : une réflexion sur des concepts ou une conceptualisation assumée des notions juridiques étudiées, un usage de la théorie du droit et le recours à des disciplines auxiliaires, la philosophie en tout premier lieu, mais également la linguistique ou la logique ${ }^{6}$. Dans un tel contexte, qui ne concerne certes encore que la jeune, en âge, doctrine constitutionnelle française, il n'est peut-être pas inopportun de penser que le contexte contemporain de la recherche en droit invite à un moment épistémologique, susceptible d'ailleurs de dépasser le seul discours constitutionnel car il concerne toute la discipline juridique. L'ambition scientifique de la jeune recherche doit s'accompagner d'un mouvement de fond visant à mettre en évidence auprès de tous les juristes le caractère décisif d'une réflexion sur le cadre conceptuel et méthodologique de son discours. Le terme est galvaudé, suremployé et il représente plus qu'il ne fait sens, mais il s'agit bien ici de s'inscrire dans une démarche réflexive sur ce que c'est que «faire du droit». L'enjeu est d'autant plus décisif qu'en améliorant la qualité de son discours, le juriste pourra d'autant mieux prétendre à disposer d'une place et d'une visibilité vis-à-vis des autres disciplines scientifiques et en particulier des autres sciences sociales. L'enjeu n'est pas seulement institutionnel, il est surtout une condition du dialogue avec les autres sciences, ce dialogue étant à son tour susceptible de nourrir la réflexion du juriste sur lui-même. Il faut pouvoir clairement situer ce que l'on fait et comment on le fait pour pouvoir entreprendre un dialogue scientifique aussi bien avec les membres de sa propre communauté scientifique qu'avec les autres disciplines. Poser son cadre conceptuel d'analyse est une condition première permettant la discussion scientifique. La falsification éventuelle des propositions d'analyse scientifiques n'est possible que pour autant que le cadre théorique dans lequel elles s'insèrent est connu.

Pour ouvrir la réflexion, nous ébaucherons à grands traits les différents éléments structurants d'un discours juridique scientifique et donc critique. $\mathrm{Ne}$ posant que les prémisses d'un programme à partir de réflexions relativement générales et en s'inscrivant dans une démarche mettant en lumière les conséquences pour le droit de questions épistémologiques d’ordre général, nous nous contenterons d'exposer ce qui semble décisif pour le discours sur le droit d'un point de vue épistémologique, sans forcément les rattacher à des références générales en épistémologie. Pour poser les jalons de ce programme, il semble nécessaire de s'intéresser successivement à quatre questions : à l'objet observé (I), au discours sur cet objet (II), au rapport entre l'objet et le discours sur cet objet, et donc à la question de

5 L'analyse mérite d'être approfondie mais il est possible de s'interroger sur le fait que la « juridicisation » du discours constitutionnel dans la lecture jurisprudentielle de son objet ait été accompagnée d'un abandon relatif des questions conceptuelles. La question mérite d'autant plus d'être posée que les cadres conceptuels classiques aujourd'hui encore mobilisés sont issus d'une doctrine du début ou du milieu du siècle dernier.

6 Sans citer ici des thèses en particulier, ce qui imposerait de les classer et de mettre en évidence leur mérites respectifs, nous conviendrons, à partir de l'expérience de la lecture des thèses actuelles, que ces qualités sont aujourd'hui acquises pour un nombre significatif d'entre elles. 
la vérification des propositions du discours sur l'objet (III) et enfin à la spécificité du discours sur le droit et à ses rapports avec les disciplines auxiliaires (IV). Il faudra sans doute envisager un cinquième point. À ce programme épistémologique individuel, il convient d'ajouter, dans le prolongement, un programme collectif et institutionnel susceptible de permettre, d'accompagner et de renforcer les conditions de son existence $(\mathrm{V})$.

Cette présentation présuppose une distinction première essentielle entre l'objet du discours et le discours sur cet objet. Le droit est un objet observable distinct du discours qui porte sur cet objet. Un énoncé constitutionnel écrit, " $X$ », dans une Constitution est un objet d'observation pour le constitutionnaliste, le discours que ce dernier produit sur cet énoncé, du type par exemple, " $X$ signifie $Y$ », est distinct de cet énoncé. L'énoncé «le mandat impératif est nul » contenu dans l'article 27 de la Constitution est distinct du discours sur cet objet qui consisterait à soutenir que « l'article 27 de la Constitution française interdit tout mandat impératif ». Cette distinction simple n'en est pas moins décisive lorsqu'il s'agit de penser les conditions de la connaissance en droit. Celles-ci concernent, en l'occurrence, le discours sur le droit et non pas le droit lui-même. Les questions qui seront successivement abordées portent sur la connaissance du droit et donc sur le discours sur le droit et non pas sur l'objet de ce discours.

\section{I.- L'OBJET}

La condition première d'un discours scientifique est de disposer d'un objet d'observation. Sans entrer dans les débats philosophiques traditionnels entre, si l'on accepte un tel raccourci, réalisme et constructivisme, il est possible de soutenir sans trop de risques que l'objet de la science est toujours un objet construit par le discours sur cet objet. S'il existe le plus souvent dans les différentes sciences quelles qu'elles soient, un objet réel à observer, aucune d'entre elle ne saurait se limiter à la seule observation immédiate de cet objet. La science a précisément pour fonction de voir au-delà de ce qui se présente au regard avec la force de l'évidence. Le boson de Higgs, par exemple, était d'abord une hypothèse insusceptible de se rattacher à une réalité observable avant, précisément, d'en devenir une, tout comme a pu l'être la théorie de l'héliocentrisme de Copernic. La démarche scientifique est précisément une démarche sceptique sur ce qui est observable, qui se doit de lutter contre les perceptions premières et les évidences immédiates sur ce qui est observé, et consiste à voir au-delà de ce qui apparaît de manière évidente. Elle oblige à une approche constructive pour établir ce qui est observé.

Pour les juristes, cette démarche constructive impose de disposer d'une théorie du droit, c'est-à-dire d'une description et d'une formalisation globale de ce qu'est et de comment fonctionne le droit en général. Ils doivent disposer d'une définition du droit susceptible de l'identifier quels que soient ses contextes d'application et expliciter comment celui-ci se structure, s'organise en général. Tel est l'objet d'une théorie générale du droit que de proposer une définition et de permettre une identification de ce qu'est le droit en général et de comment il fonctionne, indépendamment de tel ou tel autre droit positif particulier. Une fois posée, cette théorie du droit fournit le cadre conceptuel à partir duquel il sera possible d'observer tous les droits en vigueur quels qu'ils soient et, surtout, au-delà de leurs contingences et de leurs spécificités.

Le cadre conceptuel général que fournit la théorie du droit est une nécessité pour identifier, situer, catégoriser, classer et qualifier les différents objets susceptibles d'être observés par le juriste. Une théorie du droit doit permettre, en particulier, de différencier et de situer entre eux : 
- ce qui relève du droit et ce qui n'en relève pas ;

- les différents discours, à savoir les discours du langage objet, discours du législateur, du juge, et les discours sur le langage objet, de la doctrine (discours théorique et discours de systématisation du droit positif), du praticien du droit ;

- les catégories de normes et leurs rapports entre elles.

Sur le premier point, il convient de préciser que si une théorie du droit permet de distinguer ce qui relève de son objet et ce qui n'en relève pas, elle n'impose pas forcément d'exclure l'étude de qui n'est pas son objet, soit qu'il y ait précisément un intérêt à disqualifier ce qui pouvait prétendre à être objet d'analyse, soit que le lien entretenu entre ce non-objet et l'objet justifie que l'on étudie le nonobjet. Par exemple, d'un point de vue théorique, selon une perspective normativiste, le droit règle sa propre production et, pour qu'une norme soit considérée comme une norme, il faut qu'une autre norme lui donne cette qualité. La question de savoir si, dans un système donné, la jurisprudence pose des normes générales et abstraites et donc si elle pose du droit doit être résolue par la recherche de l'existence d'une norme d'habilitation en ce sens. En l'absence d'une telle norme, la jurisprudence ne sera pas considérée comme du droit ; mais, il aura été pertinent de rechercher et de montrer ce qui fait qu'elle n'est pas du droit pour clarifier son statut. Selon la même perspective théorique, l'étude de la jurisprudence peut apparaître pertinente dans un système juridique donné, dès lors que celle-ci, même si elle ne pose pas de norme générale et abstraite, participe à l'interprétation de normes générales et abstraites et, plus généralement, au processus de concrétisation du droit. Classer n’impose pas forcément d'exclure mais, surtout, de situer ce qui est observé.

La nécessité de disposer d'une théorie du droit ne saurait être discréditée par le fait que, le plus souvent, les juristes ne disposent pas d'une théorie du droit telle qu'elle a été définie. Pourtant, même sans disposer d'une théorie du droit, chaque juriste a une approche minimale et intuitive de ce qu'est son objet d'étude et donc de ce qu'est le droit. Développer un discours juridique présuppose nécessairement d'avoir un minimum de connaissances sur ce qu'il s'agit d'observer et la manière dont il convient de le faire. En réalité, chaque juriste dispose d'une théorie du droit minimale, incomplète et intuitive lui permettant de disposer d'un objet d'étude et de développer un discours sur cet objet. En outre, ceux-là mêmes qui refuseraient de se lier à un cadre conceptuel prédéterminé pour observer le droit posent déjà, en réalité, un cadre conceptuel d'analyse, précisément et non sans certaines contradictions, un cadre conceptuel sans cadre d'analyse. Ils choisissent déjà un cadre épistémologique.

Il reste que se contenter d'une préconception du droit n'est pas satisfaisant, faute de pouvoir expliquer chacun des phénomènes susceptibles d'être observé dans le droit. Une théorie du droit se doit d'être générale : elle doit identifier ce qui est du droit et ce qui n'en est pas, les rapports entre ces deux éléments, expliciter ce qui caractérise l'existence d'une norme et donc le critère de la juridicité, comment les normes s'agencent entre elles, éclairer les questions de régularité/irrégularité entre les normes... Plus le cadre conceptuel général sera élaboré, logique, complet et capable de proposer une analyse cohérente de chaque situation concrète susceptible d'être identifiée en droit positif, plus l'acuité de la vision sur son objet sera importante.

Une dernière confusion mérite que l'on s'y attarde quant au choix de son objet et donc de sa théorie du droit. La critique du subjectivisme peut se faire jour et, par-là, la négation du caractère scientifique de la démarche théorique. Il y aurait toujours une part de subjectivité dans le choix d'une théorie du droit, ce qui peut conduire à refuser de trancher entre une théorie plutôt qu'une autre et/ou à emprunter à plusieurs théories les explications susceptibles d'être apportées à une 
question particulière. Cette subjectivité témoignerait, par ailleurs, d'un relativisme des théories du droit et donc d'un caractère non-scientifique de ces dernières. Cette critique repose sur une approche simpliste et idéalisée des sciences dures. Seules ces dernières pourraient prétendre à une totale neutralité de par leur capacité à révéler le vrai. Si poser un cadre conceptuel implique de choisir, c'est également un moyen d'être vu, lu et critiqué à partir de ce cadre conceptuel et donc de favoriser la discussion scientifique. Ce n'est que par ce moyen qu'il est possible de mieux connâitre en choisissant les cadres à partir desquels un objet sera observé. Les choix conceptuels ne sont pas figés et c'est précisément la discussion scientifique qui permet de les adapter afin de rendre possible une meilleure connaissance des objets décrits et se rapprocher ainsi d'une certaine vérité à un moment donné. Même en sciences sociales, l'objectif de la recherche est de se rapprocher de la vérité, c'est-àdire de la meilleure manière de voir les choses pour révéler ce qu'elles sont. Sans cadre, sans choix, point de discussion et point de recherche d'une meilleure connaissance. Le choix méthodologique ne saurait être réduit à une question de points de vue relatifs et à la subjectivité. Il est un choix premier et nécessaire pour conduire une démarche scientifique.

De plus, le mythe d'un usage pluraliste des théories du droit est inconcevable dès lors qu'une théorie se veut générale et qu'elle entend fournir un cadre d'analyse de toutes les situations susceptibles d'être observées en droit positif. Une même situation peut faire l'objet de deux propositions d'analyse théoriques différentes, mais chacune d'entre-elle ne sera applicable en principe qu'à l'exclusion de l'autre ou une seule d'entre-elle pourra prendre sa place dans une théorie plus globale à l'exclusion de l'autre. Sur la question de savoir qui est, par exemple, le producteur des normes constitutionnelles dans un système donné, l'on retiendra, de manière simplifiée, que le normativisme considère que ce sont le pouvoir constituant et le pouvoir de révision constitutionnelle qui produisent de telles normes, alors que le réalisme soutient que c'est le juge constitutionnel, en tant qu'interprète authentique du texte constitutionnel, qui pose des normes constitutionnelles. Ces deux explications théoriques ne peuvent être simultanément retenues sous peine d'entrer en contradiction. De plus, chacune de ces deux explications n'est cohérente que dans le cadre théorique général dans lequel elle s'insère, le normativisme ou le réalisme, et ne saurait s'intégrer dans l'explication théorique concurrente.

Disposer d'un cadre conceptuel général à partir duquel il est possible d'observer le droit est nécessaire, mais n'est pas suffisant. Le discours sur le droit, et la théorie du droit en est en l'occurrence un, doit présenter certaines qualités.

\section{II.- LE DISCOURS SUR L'OBJET}

Pour développer un discours scientifique, plusieurs éléments doivent être mis en évidence. Le premier d'entre eux est sans doute celui de la mise à distance $d u$ discours sur l'objet vis-à-vis de l'objet observé. Le discours ne saurait être une simple répétition de l'objet observé, mais un regard distancié et critique susceptible de mettre évidence tout ce que cet objet ne dévoile pas de manière immédiate. L'objectif est de mieux connaître ce qui est décrit et non pas de le répéter en usant d'un langage synonymique. Aussi convient-il pour le discours sur l'objet de ne pas se contenter de reprendre le discours du langage objet. Il ne suffit pas de répéter les énoncés du langage objet pour les connaître ; il faut les soumettre à l'épreuve de la conceptualisation pour en éprouver et en construire le sens. La question de l'apport à la connaissance du discours du juriste est au cœur de l'ambition scientifique de celuici. Par exemple, identifier l'usage de ce qui est qualifié de «contrôle de proportionnalité » par le Conseil constitutionnel ne saurait se limiter à l'étude des 
seules décisions de celui-ci dans lesquelles il utilise l'expression. Une telle orientation exige de poser un concept général de «contrôle de proportionnalité » permettant, de manière autonome vis-à-vis du langage objet, d'identifier un tel contrôle. Autrement dit, la question des définitions est décisive et impose une construction distanciée de celles-ci par rapport à ce que ces définitions sont censées décrire. Il n'est possible de valablement observer le « contrôle de proportionnalité » dans la jurisprudence du Conseil constitutionnel que si l'on a retenu une définition autonome de cette expression.

Cette réflexion doit par ailleurs être mise en perspective avec un autre élément tout aussi décisif que doit revêtir le discours : son caractère descriptif. Cette exigence impose que le discours sur le droit se contente de décrire ce qu'il est sans jamais prescrire ce qu'il doit être. Le discours doit ainsi s'imposer une neutralité, en termes de jugement de valeur, vis-à-vis de son objet, même si celui-ci, le droit, e60t à lui, empreint de valeurs. Il est possible de commenter la loi sur le mariage pour tous, qui véhicule certaines valeurs sur l'institution du mariage, sans émettre aucun jugement de valeur sur celle-ci. Le caractère descriptif du discours véhicule un certain nombre de malentendus tendant à lui dénier toute dimension critique. Décrire consisterait simplement à répéter, à reproduire ; or, en réalité, décrire c'est connaître, sans juger. Dès lors que la description s'accompagne d'une mise en distance de l'objet, elle permet de révéler ce qui n'apparaît pas au regard de manière évidente ou ce qui est masqué. Elle présuppose donc un regard critique c'est-à-dire un regard qui ne se contente pas d'une appréhension première et immédiate de l'objet observé. Ce n'est pas la description qui en elle-même ne serait d'aucun apport cognitif, mais bien le manque d'ambition de la description et une approche restrictive de ce qu'est la connaissance. Toute étude qui se contente de reproduire, par exemple, le discours du juge pour étudier certaines notions que celui-ci utilise, sans aucune conceptualisation de celles-ci, présente une dimension cognitive faible et sans doute même la plus faible qui soit.

La mise à distance et la description n'ont de dimension de connaissance que si elles sont au service d'un questionnement, d'une problématique. La connaissance ne se conçoit qu'à travers la formulation de questions autour desquelles un savoir peut être organisé. Le savoir ne possède de véritable valeur de connaissance que s'il est structuré autour de problématiques. La problématique apparaît ainsi comme une clé d'entrée vers le savoir à même d'offrir un meilleur regard sur ce qui est décrit. Suffitil, pour étudier la justice constitutionnelle, de connaître la composition, le fonctionnement et les compétences d'une juridiction constitutionnelle et donc se contenter du régime juridique de celle-ci ? Ne convient-il pas plutôt de situer ces informations - peut-on seulement parler de connaissances ? - dans le cadre de l'État de droit, de la démocratie et/ou du régime représentatif pour mieux en révéler le sens? Poser les questions que soulève son objet constitue le meilleur moyen de mieux le voir et donc de mieux le connaître. Tout l'enjeu de la connaissance se concentre dans la recherche des bonnes questions qui permettent d'accéder à une meilleure vision de ce qui est décrit.

Un dernier élément mérite notre attention. Le discours sur le droit doit enfin user d'un langage précis et rigoureux et les propositions soutenues doivent répondre aux exigences de la logique formelle. L'usage d'un langage précis et rigoureux n'appelle pas de précision particulière. Il est la condition première de toute analyse ayant une prétention scientifique. Concernant la logique formelle, deux principes au moins seront rappelés : le principe d'identité et le principe de non-contradiction. En vertu du premier, $P$ est $P$; en vertu du second, il n'est pas possible, de manière simultanée, que $P$ et que non- $P$. L'on pourrait ajouter le principe du tiers exclu, aujourd'hui discuté dans son autonomie vis-à-vis des deux autres, en vertu duquel $P$ ou non- $P$ est 
vrai, c'est-à-dire que soit $P$ est vrai, soit non- $P$ est vrai. Toute proposition est donc soit vraie, soit fausse. La théorie réaliste de l'interprétation lato sensu, intégrant la théorie des contraintes à la théorie réaliste stricto sensu, défend à la fois la liberté juridique du juge d'interpréter les énoncés normatifs ${ }^{7}$ et l'existence de contraintes juridiques pesant sur les interprètes ${ }^{8}$. Elle méconnaît ainsi à la fois le principe de non-contradiction et celui de tiers exclu?

La scientificité du discours repose alors sur la précision du langage et la logique d'ensemble des propositions d'analyse. Cet usage rigoureux du langage renvoie à la question des arguments. Le discours sur le droit est un discours qui se construit à partir d'arguments à l'appui des propositions d'analyse avancées. De là, la qualité du discours dépend de la qualité de l'argumentation et donc de la logique et de la précision du langage. Toute la qualité du discours est donc interne à celui-ci, quels que soient les arguments utilisés. Même un argument de type empirique, reposant par exemple sur des statistiques, ne sera déterminant que s'il présente une logique interne satisfaisante. Pour le dire autrement, même un argument empirique, peut être un mauvais argument s'il est mal utilisé.

Cette présentation des qualités du discours se doit d'être illustrée par des exemples tirés des deux types de discours sur le droit susceptible d'être tenus, le discours de théorie du droit, dont nous avons déjà indiqué ce qu'il était, et le discours de dogmatique juridique qui entend analyser et systématiser le droit positif. Ces deux discours ne sauraient être hiérarchisés car ils n'ont pas le même objet et s'appuient tous deux sur une dimension conceptuelle, ce qui entretient d'ailleurs parfois des confusions sur l'apport «théorique » ou sur ce que c'est que «faire de la théorie ». Si « faire de la théorie » consiste à systématiser et à proposer des concepts explicatifs, la théorie du droit comme la dogmatique «théorisent». Tel n'est cependant pas l'élément que nous avons retenu pour distinguer ces deux types de discours; le discours théorique identifie le droit en général, la dogmatique juridique le droit positif. À la rigueur, pourraient être distinguées des théories du droit positif et des théories du droit en général. Tout est ensuite question de seuil pour déterminer si un concept reflète le droit en général ou le droit positif en particulier. La question peut se poser à partir des trois éléments classiques composant le «droit de propriété », pour caractériser le lien existant entre le titulaire de ce droit et la chose qui en fait l'objet, à savoir l'usus, l'abusus et le fructus : participent-ils d'une théorie du droit en général ou d'une théorie du droit positif ? La conceptualisation du droit de propriété est nécessaire ; la question de savoir si cette conceptualisation relève d'une théorie générale du droit ou d'une théorie du droit positif demeure difficile à trancher. $A$ priori, il s'agit d'une conceptualisation d'un droit particulier et non pas celle du droit en général.

Reste à illustrer les exigences mises en évidence et pesant sur le discours juridique sur chacun des deux types de discours. La théorie du droit ne semble pas poser de difficultés. Elle présuppose d'emblée une mise à distance de son objet, puisqu'elle entend précisément le construire en général. Elle vise par ailleurs

7 Pour une affirmation en ce sens : M. TROPER, «Une théorie réaliste de l'interprétation », in $L a$ théorie du droit, le droit, l'État, Puf, Leviathan, 2001, p. 84 ; "La liberté d'interprétation du juge constitutionnel », in Interprétation et droit, sous la direction de P. Amselek, Bruylant, 1995, p. 241.

8 Pour une affirmation en ce sens selon une formulation des plus récentes visant à mettre en évidence les contraintes «spécifiquement juridiques » qui pèsent sur «l'acteur juridique » : V. CHAMPEILDESPlATS, M. TROPER, «Proposition pour une théorie des contraintes juridiques", in Théorie des contraintes juridiques, Bruylant LGDJ, La pensée juridique, 2005, p. 11.

9 Sur les critiques du réalisme et les références bibliographiques sur cette question, voir : Théorie(s) $d u$ droit, Ellipses, Manuel Universités Droit, 2008, p. 149 et s. Voir également : E. PICARD, « Contre la Théorie réaliste de l'interprétation juridique ", Revue juridique de l'USEK, n 10, 2009, pp. 21 110 . 
seulement à décrire ce qu'est le droit et comment il fonctionne en général ; elle n'entend pas dire comme il doit ou il devrait être. Elle doit mettre en évidence les grandes questions déjà évoquées que soulève le droit : qu'est-ce que le droit ? Quel est le critère de la juridicité ? Comment s'organisent les normes entre elles... ? Les propositions d'analyse doivent répondre aux principes de la logique formelle pour constituer un ensemble descriptif cohérent du droit. Les arguments sur lesquels doit s'appuyer la théorie peuvent reposer sur des illustrations de droit positif pour montrer en quoi les propositions théoriques permettent de les éclairer et de mieux les comprendre. Ils doivent, par l'illustration, emporter la conviction quant à la capacité explicative de la théorie retenue.

L'illustration de ces exigences avec le discours dogmatique est sans doute moins évidente à proposer et elle n'en est pas moins décisive. Elle mérite de s'appuyer sur ce qui peut apparaître le niveau le plus bas de l'exercice traditionnel auquel est confrontée la dogmatique : le commentaire de jurisprudence et, pour ce qui nous intéresse plus particulièrement, celui d'une décision du juge constitutionnel. Dans cet exercice, le juriste s'appuiera, en tout premier lieu, sur une théorie du droit qui lui permettra de situer le statut de la jurisprudence (s'agit-il de normes, de normes juridiques, de quelles normes juridiques ?), la manière dont elle se situe dans le processus juridique (est-elle le droit, une concrétisation ou une application du droit ?), la place des normes juridiques de référence dans cette décision (caractère contraignant ou non contraignant de ces normes juridiques?), ce que les visas, les motifs et le dispositif de la décision de justice formalisent dans le processus juridique. Le commentateur dispose d'un cadre conceptuel permettant d'identifier clairement son objet et de le catégoriser. Cette grille de lecture n'est toutefois qu'un prérequis minimum qui situe l'objet à décrire, mais qui ne participe pas encore de sa description. Un autre prérequis concerne la connaissance du droit positif, c'est-à-dire des normes de référence qui sont utilisées par le juge, en ellesmêmes et au regard des différentes interprétations qu'elles sont susceptibles de receler et, plus largement, des autres décisions rendues précédemment et portant sur des affaires similaires, qu'elles concernent la norme contrôlée ou les normes de référence du contrôle. La contextualisation de la décision commentée est nécessaire. Il convient encore de mettre à distance les éléments contenus dans la décision grâce à l'utilisation de concepts descriptifs susceptibles de situer les notions utilisées par le juge : droits fondamentaux, contrôle de proportionnalité, incompétence négative, les garanties légales des exigences constitutionnelles... La lecture d'une décision mobilise ainsi un ensemble de connaissances à divers niveaux. Ces éléments connus, il convient d'identifier la question, le problème juridique soulevé par la décision à partir duquel il s'agira de décrire la décision rendue. Cette problématique permet de porter un point de vue spécifique sur la décision et de mettre en évidence sa signification et son apport. Le commentaire n'est déjà plus ici une simple reproduction du langage du juge, mais une problématisation de la décision qu'il rend. Dans la décision, l'attitude du juge devra être décrite pour en établir le sens et pourra ensuite être évaluée à partir de toutes les connaissances requises pour sa lecture et, en particulier, des connaissances conceptuelles qui appellent l'usage d'un langage précis et imposent de poser des définitions. Cette évaluation permet de mettre en évidence ce qui est satisfaisant ou ce qui ne l'est pas au regard du cadre général d'analyse et implique donc le développement de propositions alternatives en conformité avec le cadre conceptuel d'analyse. Ainsi, par exemple, lorsque l'on souscrit à une analyse normativiste, il s'agira de vérifier, dans le système étudié, la régularité de la concrétisation normative faite par le juge au regard de la norme générale et abstraite telle qu'elle a été voulue par son auteur et d'envisager d'autres concrétisations possibles et donc d'éventuelles autres interprétations possibles. Il sera 
ensuite nécessaire d'évaluer entre eux ces différents possibles mis en évidence. La démarche explicative et critique doit être logique et s'appuyer sur des arguments à l'appui de chacune des propositions d'analyse formulées. Le commentaire-description de la décision n'a alors rien d'une simple répétition du discours du juge. Il implique une mise en perspective critique de celui-ci pour mieux le connaître et en révéler le sens. Révéler ce qui n'est pas dans la décision pour mieux connaître celle-ci, tel pourrait être le résumé-slogan de ce que doit être un commentaire de décision.

Ce que doit être le discours sur le droit pour prétendre à un minimum de scientificité n'épuise pas cette question de la scientificité du discours. Dans un contexte de domination des sciences dures comme image et modèle même de ce que doit être la science, la question de la vérification des propositions formulées par le discours sur le droit devient cruciale.

\section{III.- LA VÉRIFICATION DES PROPOSITIONS DU DISCOURS SUR L'OBJET}

La question de la vérification des propositions émises par le discours scientifique est décisive et elle n'est pas sans poser des difficultés considérables dans les sciences en général et dans les sciences sociales en particulier. De manière schématique, deux modèles s'opposent quant aux possibilités de vérification des propositions scientifiques: un modèle empirique et un modèle analytique. Le modèle empirique suppose que toutes les propositions émises doivent, pour être vraies, pouvoir être vérifiées par l'expérience en s'appuyant sur des faits réels objectivement observables. Le modèle analytique considère que la véracité des propositions dépend de la qualité et de la précision du langage utilisé pour décrire un objet et du respect par les propositions d'analyse des principes de la logique formelle. Le modèle empirique renvoie à l'observation d'un élément extérieur à la proposition scientifique pour vérifier qu'elle est vraie ou qu'elle est fausse; le modèle analytique, à la cohérence interne de cette même proposition. La discipline juridique est traversée par ces deux modèles et l'on retrouve, derrière chacun d'entre eux, en substance, l'opposition entre réalisme et constructivisme.

Il reste que l'opposition empirique/analytique ne saurait être trop simplificatrice. Du côté de l'empirisme, il peut exister des propositions scientifiques vraies insusceptibles de vérification empirique au moment où elles sont émises et qui pourront l'être seulement par la suite, en particulier lorsque les techniques d'observation du réel seront en mesure de le permettre. Il reste, qu'au moment où elles sont émises, ces propositions ne sont que des hypothèses et elles n'en sont pas moins vraies alors qu'il est impossible de les vérifier de manière empirique. La preuve par l'observation demeure en tout état de cause relative à partir du moment où tout n'est pas visible ou ce qui est visible peut tromper sur ce qui est réellement. Pour prendre un exemple caricatural, l'on peut, à l'œil nu, tenter de vérifier l'existence des atomes qui composent la tasse à café posée sur un bureau, il sera vain, en l'absence de superpouvoir de vision, d'être en mesure de prouver quoi que ce soit par la seule observation de la réalité qui se révèle à l'œil nu. Du côté de l'analytique, la construction d'un objet à observer ne se fait pas en dehors de toute réalité observée. Une démarche analytique n'est pas hors sol. Elle tend seulement à construire un objet à partir de l'observation d'une réalité, mais en veillant à mettre en évidence l'essence même de celle-ci susceptible de mieux l'observer et donc de mieux la connaître. Plus concrètement, la construction de l'objet droit ne saurait se faire sans observer ce qu'est le droit ou, du moins, ce qui est appelé droit, mais il s'agit de prendre distance avec cet objet pour pouvoir en proposer une définition générale, au-delà des contingences, à même de l'identifier, quels que soient les différents droits observés. 
Ces rapides nuances exposées tendent, en définitive, à mesurer de manière relative la possibilité de vérifier les propositions d'analyse émises. Au-delà de toute vérification empirique ou de solidité interne d'un discours analytique, c'est sans doute la capacité d'une proposition scientifique à mieux connaître l'objet qu'elle décrit. L'apport cognitif d'une proposition et sa capacité à s'insérer dans un ensemble cohérent de propositions à même d'analyser de manière générale l'objet décrit permettent d'apprécier de manière qualitative un discours scientifique. Ce sont donc moins, peut-être et dans une certaine mesure, la vérification des propositions d'analyse ou leur logique et leur cohérence interne qui importe que l'apport à la connaissance qu'elles recèlent dans la description des objets qu'elles entendent observer.

Il reste, qu'entre les deux modèles, il est nécessaire de choisir. Il n'est pas possible de s'inscrire à la fois dans une logique empirique et une logique analytique, particulièrement en droit. S'inscrire dans une démarche empirique imposera d'envisager le droit comme un fait, les propositions sur le droit étant susceptibles d'être vérifiées à partir de faits réels. Cette question de choix renvoie plus largement à celle de la spécificité du discours juridique et donc à son rapport avec les autres sciences.

\section{IV.- LA SPÉCIFICITÉ DU DISCOURS JURIDIQUE ET LES SCIENCES AUXILIAIRES}

La question du choix du modèle de vérification des propositions d'analyse de la science juridique emporte des conséquences sur la spécificité du discours juridique tant dans son objet que dans ses méthodes, ce qui implique de le situer par rapport aux sciences auxiliaires ${ }^{10}$.

En s'inscrivant dans une logique factuelle, le discours juridique se doit alors d'utiliser les méthodes d'analyse des sciences empiriques ou de type empirique. L'observation de terrain, l'élaboration de questionnaires, les statistiques, la modélisation mathématique seront autant de méthodes à emprunter pour une approche factuelle du droit. Il appartient alors au discours juridique d'emprunter les méthodes aux autres disciplines empiriques pour proposer un discours vérifonctionnel. La spécificité du discours juridique devient toute relative à défaut de disposer d'un objet et d'une méthode spécifique. Le discours juridique se fond dans les disciplines auxiliaires. Il n'en garde pas moins une identité minimale dans la mesure où il doit, du moins le suppose-t-on, se réapproprier les méthodes pour les adapter à la singularité des questions propres à son objet. L'on pourrait cependant voir là un certain paradoxe, en l'absence de spécificité de son objet, à soutenir une spécificité de ses méthodes ou, au minimum, de son regard sur son objet d'étude.

Dans une logique de type analytique, le discours juridique construit son objet indépendamment de toute vérification empirique et peut ainsi mettre en évidence la spécificité de son objet. Le discours juridique est en mesure de disposer d'un objet singulier. Par exemple, considérer que son objet est constitué de l'étude de la signification d'énoncés prescriptifs, qui établissent ce qui doit être et non ce qui est, marque non seulement une singularité de son objet, mais également une spécificité de celui-ci par rapport aux objets factuels d'autres sciences sociales. De plus, cette construction de l'objet dans le devoir être éloigne également le discours juridique du discours des sciences naturelles. Alors que ces dernières sont gouvernées

10 L'expression est empruntée à R. Ponsard qui l'a repris de J. Le GOFF, Les catégories juridiques et le Conseil constitutionnel. Contribution à l'analyse du droit et du contentieux constitutionnel, op. cit., note 340 , p. 189. 
par le principe de causalité, qui veut que lorsque des conditions sont réunies alors une conséquence se produit nécessairement, le premier est dominé par le principe d'imputation, à savoir que si des conditions sont réunies alors quelque chose doit se produire, sans que l'on sache si ce quelque chose se produira ou ne se produira pas. Une telle analyse de type positiviste garantit une spécificité de l'objet du juriste et donc une autonomie disciplinaire du côté de l'objet observé. La spécificité de l'objet ne saurait pour autant conduire à un isolement méthodologique et à une autarcie disciplinaire ${ }^{11}$. L'étude du droit en général, la théorie du droit, et l'étude du droit positif en particulier, la dogmatique juridique, soulèvent différentes questions susceptibles d'être éclairées par des disciplines auxiliaires. Elles soulèvent des questions d'interprétation et doivent, en conséquence, mobiliser des connaissances en linguistique et en sémiotique, de logique et recourir à la discipline du même nom; le droit est l'expression du pouvoir et la sociologie et la science politique pourront être éclairantes; il est empreint de valeurs et mobilise des concepts explicatifs, la philosophie pourra être utile. L'objet étudié est spécifique. Les méthodes empruntent aux autres disciplines. Pour être plus précis, doivent être utilisées les méthodes proposées par les disciplines qui ont précisément pour objet ce que la discipline juridique recherche à faire ; aussi l'interprétation d'un énoncé pourra-t-elle faire appel à la linguistique. Cet emprunt méthodologique doit être adapté à la spécificité de son objet et impose donc, au-delà de l'emprunt, une réappropriation méthodologique.

Le recours aux disciplines auxiliaires doit permettre une meilleure connaissance de l'objet droit tel qu'il a été construit. Il ne s'agit pas de faire de la sociologie pour produire un savoir scientifique dans cette discipline mais de recourir aux travaux de sociologie existants pour en dégager des éléments éclairants pour la connaissance du droit. C'est ce que défend en l'occurrence Régis Ponsard avec l'approche pluridimensionnelle du droit qui est une approche juridique pluridimensionnelle ${ }^{12}$, et c'est ce qui a été également soutenu, sans que les deux approches ne soient pour autant les mêmes, avec la méthode transdisciplinaire normativo-centrée ${ }^{13}$. Reste à préciser ce qu'il faut entendre, en pratique, par « recours » aux autres sciences. L'emprunt méthodologique a déjà été évoqué, la question de l'usage des résultats d'analyses proposées par d'autres sciences demeure. Il est possible de recourir seul et de manière autonome aux résultats produits par d'autres sciences et de s'en nourrir de manière libre. Une telle démarche, pour être scientifiquement pertinente, ne saurait être conduite sans disposer d'un minimum de connaissances sur la ou les disciplines auxiliaires auxquelles l'on a recours, ne serait-ce que pour pouvoir apprécier de manière critique les travaux utilisés, les situer dans le contexte de la discipline, dans les différents courants, les différentes écoles et les évaluer dans un contexte d'ensemble. Là encore, rien n'empêche d'être autodidacte, seul le temps à consacrer risque d'être limité pour peu que l'on demeure spécialiste de sa discipline première, le droit, d'autant plus que les contraintes extérieures à la recherche s'accroissent aujourd'hui. L'argument est d'ordre pratique ; il n'en révèle pas moins les limites à une démarche trop ambitieuse. Rien n'empêche cependant, c'est une autre voie à entreprendre, de former les juristes à ces disciplines auxiliaires dans le cursus universitaire, en licence, en master et/ou en doctorat, et même en formation

11 Voir sur cette expression : R. PONSARD, op. cit., p. 182 et s.

12 Sur cette approche, voir en particulier R. PONSARD, op. cit., p. 97 et s.

13 Voir : "Appréhender le droit constitutionnel jurisprudentiel sous un angle politique. D'une posture à la discussion de quelques orientations méthodologiques fondamentales ", in Questions sur la question (QsQ 3): de nouveaux équilibres institutionnels?, sous la direction de X. MAGNON, P. EsPlugas, W. MASTOR et S. MOUTON, LGDJ-Lextenso, collection Grands Colloques, 2014 , pp. 3-14. 
continue. Les obstacles institutionnels sont forts et seule une politique ambitieuse permettrait de s'inscrire dans cette voie. La voie la plus immédiatement applicable, et qui participe d'ailleurs déjà d'une formation aux autres sciences, consiste précisément à travailler avec les chercheurs des autres disciplines. Tels sont sans doute les prolongements à envisager de ce programme épistémologique.

\section{V.- PROLONGEMENT : LES CONDITIONS INSTITUTIONNELLES ET COLLECTIVES D'UNE SCIENCE DU DROIT AMBITIEUSE}

Le programme ainsi esquissé pourrait être qualifié de révolution culturelle ; celle-ci ne saurait s'imposer que par un accompagnement institutionnel à plusieurs niveaux. C'est d'abord par la formation qu'il convient d'agir. Des cours en épistémologie (juridique) et en théorie du droit sont sans doute nécessaires dès la première année de licence. Au cours de cette même année, une initiation à la plupart des disciplines auxiliaires permettant de mettre en évidence ce qu'elles sont, quel est leur objet, leur méthode et leur(s) apport(s) possible(s) à la science juridique. Sur cette question, l'enjeu institutionnel se doit d'être apprécié dans le contexte concurrentiel existant entre les Facultés de droit et les instituts d'études politiques. Les premières souffrent sans doute trop, contrairement aux seconds, d'un manque d'ouverture sur les autres disciplines. Pour tous ceux qui n'auraient pas reçu les enseignements préconisés et même pour ceux qui les auraient suivis, il faut encore en prévoir durant la formation doctorale et au titre de la formation continue des enseignants-chercheurs. L'enseignement du droit lui-même se doit d'être amélioré. La pédagogie n'impose pas une simplification excessive et une simple restitution organisée d'informations, mais plutôt une problématisation des cours d'amphithéâtre. Elle passe précisément par la mise en évidence des questions qui sont soulevées par la connaissance. La problématisation permet de clairement situer la place des connaissances et de leur donner du sens. Le savoir doit être organisé et mobilisé autour des grandes questions soulevées par les disciplines juridiques. Chaque cours, et a fortiori chaque écrit doctrinal, doit poser ses présupposés méthodologiques, ce qui participe d'une prise de conscience généralisée des questions épistémologiques. À l'évidence, cette ambition scientifique, qui impose de disposer d'un cadre conceptuel d'analyse, d'une théorie du droit, d'une méthode et de problématiser le savoir, doit encore se vérifier dans les travaux scientifiques. L'évaluation des travaux en fonction de leur apport scientifique à la connaissance et la valorisation de tels travaux permettront d'imposer cette ambition à différents moments de la vie universitaire : lors des soutenances de thèse, dans la sélection des articles publiés dans les revues comme des ouvrages publiés, par les évaluations au sein des instances nationales au moment de la qualification des maîtres de conférences et des professeurs, l'avancement de carrière et l'attribution des primes d'excellence scientifique et le recrutement de l'ensemble des enseignants-chercheurs.

La défense d'un moment épistémologique ne s'épuise à l'évidence pas avec ce dossier. La réflexion n'est qu'à peine ouverte et les pistes de réflexion seulement brièvement esquissées. Elle se poursuivra à Toulouse à l'occasion d'un colloque intitulé Quelles doctrines constitutionnelles aujourd'bui, pour quel(s) droit(s) constitutionnel(s) demain? Celui-ci a pour ambition de mettre en évidence l'utilité des théories du droit pour la connaissance du droit en général, d'expliciter la théorie du droit et les méthodes des différentes écoles de droit constitutionnel et de réfléchir sur les évolutions possibles de la doctrine constitutionnelle. Elle sera encore menée au sein de la Société européenne d'épistémologie juridique appliquée. Elle appelle plus généralement une prise de conscience collective des juristes et une discussion scientifique la plus large qui soit. La réflexion se poursuit. 